\title{
Implementation of an intelligent SINS navigator based on ANFIS
}

\begin{abstract}
In this work an intelligent navigator developed to overcome the limitations of existing Strapdown Inertial Navigation Systems (SINS) algorithm. This system is based on Adaptive Neuro-Fuzzy Inference System (ANFIS).

As in previous work, which is based on Artificial Neural Network, the window based weight updating strategy was used, and the intelligent navigator evaluated using several SINS hypothetical field tests data. And the results show that the intelligent navigator based on ANFIS more powerful compared with other (traditional and intelligent navigator based on ANN).
\end{abstract}

Keyword: Inertial navigation systems; Adaptive fuzzy system; Intelligent navigator 